DOI: https://doi.org/10.36910/6775-2524-0560-2021-44-04

УДК 681.5.

Лабжинський Володимир Анатолійович, к.т.н, доцент

https://orcid.org/0000-0003-0970-770X

Національний технічний університет України "Київський політехнічний інститут ім. Ігоря Сікорського"

\title{
ІДЕНТИФІКАЦІЯ ТИПУ АВАРІЇ НА ОБ'ЄКТАХ КРИТИЧНОЇ ІНФРАСТРУКТУРИ ЗА ДОПОМОГОЮ ПРИХОВАНИХ МОДЕЛЕЙ МАРКОВА
}

Лабжинський В. А. Ідентифікація типу аварії на об'єктах критичної інфраструктури за допомогою прихованих моделей Маркова. Розглянуто сучасні методи розпізнавання аномальних патернів у зразках кодових послідовностей для ідентифікації типу аварії на об'єктах критичної інфраструктури за допомогою прихованих марковських моделей. Представлена комплексна методика, що базується на аналізі функції густини ймовірності аномальних паттернів, що застосовується у прихованій марковській та напівмарковській моделі. Вказано, що зазначений підхід дозволяє визначити функцію часової залежності рядів даних, відповідно класифікація проводиться на основі наборів аномальних даних, що не відповідають класам навчальної вибірки. На основі математичної моделі показано, що представлена методика надає можливість оптимізувати ефективність розпізнавання аномальних паттернів при ідентифікації аварійного стану на об'єкті інфраструктури.

Ключові слова: об’єкти критичної інфраструктури, аварійний стан, аномальні патерни, функція густини ймовірності,прихована марковська модель, прихована полумарковська модель, змішана модель Гауса.

Лабжинский В. А. Идентификация типа аварии на объектах критической инфраструктуры с помощью скрытых моделей Маркова. Рассмотрены современные методы распознавания аномальных паттернов в образцах кодовых последовательностей для идентификации типа аварии на объектах критической инфраструктуры с помощью скрытых марковских моделей. Представлена комплексная методика, основанная на анализе функции плотности вероятности аномальных паттернов, котораябазируется на скрытой марковской и полумарковской модели. Указано, что указанный подход позволяет определить функцию временной зависимости рядов данных, соответственно классификация проводится на основе наборов аномальных данных не соответствующих классам обучающей выборки. На основе математической модели показано, что представленная методика позволяет оптимизировать эффективность распознавания аномальных паттернов при идентификации аварийного состояния на объекте инфраструктуры.

Ключевые слова: объекты критической инфраструктуры, аварийное состояние, аномальные паттерны, функция плотности вероятности, скрытая марковская модель, скрытая полумарковская модель, смешанная модель Гаусса.

Labzhynskyi V. A. Identification of the accidenttype at critical infrastructure facilities using hidden Markov models. Modern methods of recognizing anomalous patterns in samples of code sequences for identifying the type of accident at critical infrastructure facilities using hidden Markov models are considered. A complex technique based on the analysis of the probability density function of anomalous patterns, which is based on a hidden Markov and semi-Markov model, is presented. It is indicated that this approach makes it possible to determine the function of the time dependence of the data series; accordingly, the classification is carried out on the basis of sets of anomalous data that do not correspond to the classes of the training sample. On the basis of a mathematical model, it is shown that the presented methodology makes it possible to optimize the efficiency of recognizing anomalous patterns when identifying an emergency state at an infrastructure facility.

Key words: critical infrastructure objects, emergency state, anomalous patterns, probability density function, hidden Markov model, hidden semi-Markov model, mixed Gauss model.

\section{Вступ.}

На сьогоднішній день концепція переходу від протоколів взаємодії «людина-машина» (Person-toMachine, P2M) до протоколів взаємодії «машина-машина» (Machine-to-Machine, P2M), а також вдосконалення інтерфейсів у рамках оптимізації Р2М-взаємодії значним чином реалізується шляхом впровадження систем машинного аналізу на базі статистичних моделей, зокрема прихованих марковських моделей (Hidden Markov Model, HMM) і прихованих напівмарковських моделей (Нidden Semi-Markov Model, HSMM). Вирішення поставленої задачі є надзвичайно важливим в області науки, медицини та економічного і державного регулювання, але при цьому також слід зазначити, що зазначені дослідження найбільш актуальні при ідентифікації типу аварії на об'єктах критичної інфраструктури, де необхідно максимально знизити вірогідність похибки внаслідок «людського фактору». Очевидні обмеження, що мають бути вказані при формулюванні завдання прогнозування i класифікації аварійних станів, полягають у необхідності аналізу великих масивів даних, що надходять від вузлів сенсорної мережі, у режимі реального часу. Це зумовлює необхідність побудови адекватного математичногоапарату на основі статистичних моделей, що дозволяє сформулювати задачу оптимізації системи машинного аналізу аномальних паттернів до задачі пошуку цільових 
функцій ефективності класифікації відповідно обмежень на час обробки вхідних даних та обчислювальний ресурс апаратної платформи.

Аналіз сучасних досліджень $\boldsymbol{i}$ публікацій присвячених проблемам адаптації НММ і НSMМ при розробці систем машинного аналізу часових рядів даних у режимі реального часу вказав на актуальність робіт у галузі моделювання медичних інтерфейсів [1-3] та систем відеореєстрації [4-6].Результати дослідження показують кардинальне збільшення точності машинного аналізу при визначенні структури часових рядів. Перевага застосування у системі машинного аналізу однієї або декількох HMM (HSMM) полягає у генерації стохастичного сигналу і надає можливість побудувати універсальні та, водночас, масштабовані алгоритми класифікації на основі змішаної моделі Гаyca(Gaussian Mixture Model,GMM), що характеризуються високою точністю класифікації аномальних паттернів $[1,4,7]$. Основною задачею при цьому є оптимізація роботи алгоритму на етапі виділення викидів у часовому ряді кодової послідовності. Для вирішення цієї задачі було запропоновано ряд методик [8-10], як то додатково використовувати у системі машинного аналізу динамічну байєсівську мережу (Dynamic Bayesian Network, DBN), проводитикласифікацію аномальних фазових переходів, впровадити у загальну систему машину опорних векторів (support vector machine,SVM) та нелінійний регресійний аналіз (Kernel Nonlinear Regression Analysis, KNRA). Тим не менш, було вказано, що зазначені методики не дають достатньої точності при багатокласовому виділенні аномалій у зв'язку зі складністю налаштування порогових значень класифікації. Новітні підходи базуються на застосуванні двох НMМ, що генерують звичайні та аномальні кодові послідовності [11]. Для моделювання аномальних паттернів застосовується функція густини ймовірності (Probability Density Function, PDF), що надає змогу організувати алгоритми багатокласового виділення аномалій. Слід зазначити, що, тим не менш, зазначена модель малоефективна на рівні виділення мінімальних за значенням часового проміжку викидів, що розглядається як невирішена частина загального дослідження.

Метою дослідження стала побудова системи машинного аналізу на основі HSMM, що працює 3 випадковим розподілом аномальних зразків. Зазначений підхід включає використання HSMM при аналізі часової залежності блоків масиву данихдля виявлення миттєвих аномалій шляхом оцінки прихованих переходів між станами досліджуваного об'єкту інфраструктури.

\section{1. Постановка задачі виділення переходу між станами об'єкту на основі прихованої напівмарковської моделі}

На рівні базової моделі алгоритм класифікації виділяе кодові послідовності відповідно до навчального набору, що неефективно при роботі з аномальними кодовими послідовностями. При впровадженніР2М- та М2М-протоколів за наявності викидів у вхідних даних це призводить до помилок, що може бути критичним для роботи систем моніторингу стану об'єктів інфраструктури. 3 іншого боку, застосування статистичної моделі НММ як кінцевого автомата для якогостани,що характеризуються показником густини ймовірності, з'єднані первинним ланцюгом Марковає непридатним для стохастичного опису переходів стану на основі потоку вхідних даних, що надходить у режимі реального часу, тому у даному дослідження розглядається вдосконалена статистична модель на основі HSMM.

Для побудови математичної моделі машинного аналізу вхідних даних з метою виділення аномальних послідовностей на базі HSMM необхідно ввести наступні позначення:

- $\quad$ часовий ряд вхідних даних, представлений матрицеюD: $\left\{d_{t}\right\}$ набору базових елементів $d_{t}$, що ранжуються відповідно часуtнадходження до системи аналізу, де $t \in[1 ; T]$;

- $\quad P_{i}^{j}$ - густина тривалості стану (State Duration Density, SDD), як ймовірність того, що об’єкт залишиться у стані $j$ протягом часу $i \cdot \Delta T$, де $i \in[1 ; I]$, а $j \in[1 ; J]$;

- $\quad P_{j}$ - функція розподілу ймовірності початкового стануj(Initial State Distribution, ISD) для $\forall j \in$ $[1 ; J]$

- $\quad$ набір ймовірностей переходу від стану $j$ с стан $j^{\prime}$, представлений матрицею $\boldsymbol{C}:\left\{c_{j^{\prime}}^{j}\right\}$,причому у рамках математичного апарату задається $\mathrm{c}_{j}^{j}=0$ для $\forall j \in[1 ; J]$;

- $\quad$ показники $b_{j}\left(d_{t}\right)$ що визначає ймовірність послідовності $d_{t}$ у момент часу $t$;

- $\quad \boldsymbol{\vartheta}$ як повний набір параметрів HSMM, що включає у себе функцію $P_{i}^{j}$, матрицю переходів $\boldsymbol{C}$ і показники $b_{j}\left(d_{t}\right)$, а також параметри функції густини ймовірності (Probability Density Function, PDF). 
Таким чином ймовірність отримання ряду вхідних даних $D:\left\{d_{t}\right\}$ за умови $\vartheta$ розраховується як:

$$
\begin{gathered}
P(\boldsymbol{D} \mid \boldsymbol{\vartheta})=\sum_{j=1}^{J}\left(\alpha_{j}(T)\right), \\
\text { де набір коефіцієнтів }\left\{\alpha_{j}(t)\right\} \text { розраховується як } \\
\alpha_{j}(t)=\left[\sum_{j=1}^{J}\left(\sum_{i=1}^{i}\left(\alpha_{j}(t-i \cdot \Delta T) \cdot \mathrm{c}_{j^{\prime}}^{j} \cdot P_{i}^{j^{\prime}} \cdot \beta_{i}^{j^{\prime}}(t)\right)\right) \text { для } t>1 .\right. \\
P_{j^{\prime}} \cdot P_{1}^{j^{\prime}} \cdot b_{j^{\prime}}\left(d_{t}\right) \text { для } t=1
\end{gathered}
$$

У свою чергу набір коефіцієнтів $\left\{\beta_{i}^{j^{\prime}}(t)\right\}$ розраховується як:

$$
b_{j^{\prime}}\left(d_{t}\right)=\prod_{\tau=t_{0}}^{t}\left(b_{j^{\prime}}\left(d_{\tau}\right)\right) \text {, де } t_{0}=t-i \cdot \Delta T+1 \text {. }
$$

Наведений математичний апарат відповідає підкласу HSMM, що у сучасних дослідженнях [12-17] відносять до прихованої марковської моделі фіксованої тривалості (Explicit Duration Hidden Markov Model,ED-HMM).

Наступним кроком для моделювання змішаної моделі Гауса необхідно ввести наступні позначення:

- $\quad \boldsymbol{\varphi}_{\boldsymbol{k}}$ як повний набір параметрів GMM, що включає у себе набір $\left\{\kappa_{k}^{m}, \mu_{k}^{m}, \boldsymbol{M}_{k}^{m}\right\}$,де $k \in[1 ; K]$;

- $\quad$ модельний коефіцієнт $\kappa_{k}^{m}$, де $m \in\left[1 ; M_{k}\right]$ - кількість компонент GMM, причому $\kappa_{k}^{m} \in(0 ; \infty]$;

- $\quad \mu_{k}^{m}-$ середній вектор GMM;

- $\quad \boldsymbol{M}_{k}^{m}$ - коваріаційна матриця GMM, де $\boldsymbol{M}_{k}^{m}\left(\mu_{k}^{m}\right)=1$.

Відповідно, у рамках моделі GMM розподіл вірогідності вихідних даних (Output Probability Distribution, OPD):

$$
P_{k}^{O P D}\left(d_{t}, \boldsymbol{\varphi}_{\boldsymbol{k}}\right)=\sum_{m=1}^{M_{k}}\left(\kappa_{k}^{m} \cdot f_{G M M}\left(o_{t}, \mu_{k}^{m}, \boldsymbol{M}_{k}^{m}\right)\right),
$$

причому функція $f_{G M M}\left(o_{t}, \mu_{k}^{m}, \boldsymbol{M}_{k}^{m}\right)$ визначається як

$$
f_{G M M} \sim \frac{e^{q_{k}^{m}}}{\sqrt{\boldsymbol{M}_{k}^{m}}}, \text { де } q_{k}^{m}=-\frac{\left(o_{t}-\mu_{k}^{m}\right)^{\mathrm{T}} \cdot\left(\boldsymbol{M}_{k}^{m}\right)^{-1} \cdot\left(o_{t}-\mu_{k}^{m}\right)}{2} .
$$

Таким чином, $\boldsymbol{\vartheta}$ як повний набір параметрів HSMM може бути представлено як $\left\{P_{j}, \boldsymbol{C}, P_{i}^{k}, \boldsymbol{M}_{k}^{m}\right\}$ на основі якого оцінюється перехід до нового стану, що не може бути отримановнаслідок аналізучасових рядівданих. На основі $a_{i, j}$, що визначено через розрахунок правдоподібності, може бути виділено прихований стан як кодову послідовність $\boldsymbol{H}:\left\{h_{t}\right\}$, елементи якої розраховуються через наступне рівняння:

$$
h_{t}=\arg \left(\max _{j \in[1 ; j]} \alpha_{j}(t)\right)
$$

на основі якого проводиться ідентифікація типу аварії на об'єктах критичної інфраструктури. 
2. Побудова алгоритму класифікації з функцією густини ймовірності аномальної послідовності Аномальні послідовності часових рядів, що не входять у навчальні набори у рамках запропонованої моделі визначаються через PDF, що розрізняє набір класів виділених на етапі навчання станівоб'єкту інфраструктури (Learned Classes, LC) та нові класи (Unlearned Classes, UC), які, відповідно, вказують на потенційно аномальну послідовність, що може сигналізувати про аварійний стан об’єкту.

У даному дослідженні для визначення кодової послідовності до множини $\{k ; m\}$ застосовується модель класифікації «OvR» (One-versus-the-Rest), що на рівні математичної формалізації [13] виражається наступним чином:

$$
P\left(d_{t} \mid \varphi_{k}^{m}\right) \sim \frac{e^{\frac{1}{\varepsilon_{m}^{k} \cdot \epsilon_{m}^{k}}} \cdot\left(e^{q_{k}^{m}}-e^{\varepsilon_{m}^{k} \cdot q_{k}^{m}}\right)}{\sqrt{\left|\epsilon_{m}^{k} \cdot \boldsymbol{M}_{k}^{m}\right|} \cdot\left(\sqrt{\left(\varepsilon_{m}^{k}\right)^{I \cdot \Delta T}}-1\right)},
$$

де набір $\left\{\varepsilon_{m}^{k} ; \epsilon_{m}^{k}\right\} \in$ параметрами, що характеризують форму PDF.

При цьому для набору компонент функції $\left\{\varepsilon_{m}^{k} ; \epsilon_{m}^{k}\right\}$ відповідно $P\left(d_{t} \mid \varphi_{k}^{m}\right)$ можна вказати наступні обмеження:

$$
\left[\begin{array}{l}
\varepsilon_{m}^{k} \in[1, \infty) \\
\epsilon_{m}^{k} \in[0, \infty)
\end{array}, \text { причому } P\left(d_{t} \mid \varphi_{k}^{m}\right)=0 \text { при } \varepsilon_{m}^{k}=1\right.
$$

ЯкщоРDFпредставляє собою розподіл набору вхідних даних, наявність UC визначається через графік функції та набір компонент $\left\{\varepsilon_{m}^{k} ; \epsilon_{m}^{k}\right\}$. Ймовірність послідовності UC відповідає ймовірності переходу до нового стану $(J+1)$, що знаходиться поза визначеного наборуj $\in[1 ; J]$ :

$$
\begin{aligned}
& b_{j}^{J+1}\left(d_{t}\right)=\frac{\sum_{k=1}^{J}\left(\sum_{m=1}^{M_{k}}\left(f_{O v R}\left(d_{t} ; \varphi_{k}^{m}\right)\right)\right)}{\sum_{l=1}^{J}\left(M_{l}\right)}, \\
& \text { де } f_{O v R}\left(d_{t} ; \varphi_{k}^{m}\right)=\frac{e^{\frac{1}{\varepsilon_{m}^{k} \cdot \epsilon_{m}^{k}}} \cdot\left(e^{q_{k}^{m}}-e^{\varepsilon_{m}^{k} \cdot q_{k}^{m}}\right)}{\sqrt{2 \pi^{i \cdot \Delta T} \cdot\left|\epsilon_{m}^{k} \cdot \boldsymbol{M}_{k}^{m}\right|} \cdot\left(\sqrt{\left(\varepsilon_{m}^{k}\right)^{I \cdot \Delta T}}-1\right)} \text {. }
\end{aligned}
$$

На основі оптимізації набору параметрів HSMM з урахуванням стану, що відповідає UСможна провести оцінку аномальногостану та виявити аномальну послідовність за допомогою рівняння (6). Таким чином, розроблена математична модель надає можливість оптимізувати ефективність розпізнавання аномальних паттернів при ідентифікації аварійного стану на об'єктах інфраструктури.

\section{Висновки}

В результаті проведеного дослідження було розглянутометоди розпізнавання аномальних зразківкодових послідовностейз метоюкласифікації потенційної загрози на об'єктах критичної інфраструктури за допомогою прихованих напівмарковських моделей. Була представлена методика аналізу функції густини ймовірності аномальних зразків, що застосовується дляприхованої марковської моделі фіксованої тривалості. Зазначений підхід дозволяє визначити часову залежність рядів даних відповідно завданнюпошуку аномальних даних, що не відповідають класам навчальної вибірки. Представлена методика надає можливість суттєво підвищити рівень ефективності розпізнавання аномальних кодових послідовностей при ідентифікації та подальшій класифікації аварійного стану на об'єкті інфраструктури.

\section{References.}

1. Ji, Y., \&Zheng, S. (2021). Distributed Mode-Dependent Event-Triggered Passive Filtering for Flexible Manipulator with Semi-Markov Parameters. Sensors, 21(6), 2058. https://doi.org/10.3390/s21062058.

2. Cubero, S. N. (2015). A Mobile Manipulator Arm for Assisting the Frail Elderly and Infirm. Machine Vision and Mechatronics in Practice, 135-147. https://doi.org/10.1007/978-3-662-45514-2_12.

3. $\quad$ Siddique, M.N., Hossain, M.A., Alam, M.S., \&Tokhi, M.O. (2007). Hidden Markov Model based Fuzzy Controller for Flexible-link Manipulator. Advances in Climbing and Walking Robots. https://doi.org/10.1142/9789812770189_0074. 
4. Putra, P. U., Shima, K., \&Shimatani, K. (2018). Markerless Human Activity Recognition Method Based on Deep Neural Network Model Using Multiple Cameras. 2018 5th International Conference on Control, Decision and Information Technologies (CoDIT). https://doi.org/10.1109/codit.2018.8394780.

5. Luísa Gomes, A., Paixão, V., \&Gamboa, H. (2015). Human Activity Recognition Based on Novel Accelerometry Features and Hidden Markov Models Application. Proceedings of the International Conference on Bio-Inspired Systems and Signal Processing. https://doi.org/10.5220/0005215800760085.

6. Uddin, Z., \& Kim, T.S. (2011). Continuous Hidden Markov Models for Depth Map-Based Human Activity Recognition. Hidden Markov Models, Theory and Applications. https://doi.org/10.5772/14993.

7. Tao Xiang,\&Shaogang Gong. (2008). Video Behavior Profiling for Anomaly Detection. IEEE Transactions on Pattern Analysis and Machine Intelligence, 30(5), 893-908. https://doi.org/10.1109/tpami.2007.70731.

8. Shima, K., \& Aoki, T. (2014). A novel classification method with unlearned-class detection based on a gaussian mixture model. 2014 IEEE International Conference on Systems, Man, and Cybernetics (SMC). https://doi.org/10.1109/smc.2014.6974510

9. Lee, S., Shin, D., \&amp; Shin, D. (2019). Sensor-based Abnormal Behavior Detection Using Autoencoder. Proceedings of the Tenth International Symposium on Information and Communication Technology -SoICT 2019. https://doi.org/10.1145/3368926.3369661

10. Bae, J., \&Tomizuka, M. (2010). Gait Phase Analysis based on a Hidden Markov Model. IFAC Proceedings Volumes, 43(18), 746-751. https://doi.org/10.3182/20100913-3-us-2015.00014.

11. Mukaeda, T., \&Shima, K. (2017). A novel hidden Markov model-based pattern discrimination method with the anomaly detection for EMG signals. 2017 39th Annual International Conference of the IEEE Engineering in Medicine and Biology Society (EMBC). https://doi.org/10.1109/embc.2017.8036975.

12. Shun-Zheng Yu, \& Kobayashi, H. (2006). Practical implementation of an efficient forward-backward algorithm for an explicit-duration hidden Markov model. IEEE Transactions on Signal Processing, 54(5), 1947-1951. https://doi.org/10.1109/tsp.2006.872540

13. Mukaeda, T., Shima, K., Miyajima, S., Hashimoto, Y., Tanaka, T., Tani, N., \& Izumi, H. (2020). Development of an anomaly detection method with a novel hidden semi-Markov model incorporating unlearned states. 2020 IEEE/SICE International Symposium on System Integration (SII). https://doi.org/10.1109/sii46433.2020.9026303.

14. Luati, A., \&Novelli, M. (2021). Explicit-duration Hidden Markov Models for quantum state estimation. Computational Statistics \& Data Analysis, 158, 107183. https://doi.org/10.1016/j.csda.2021.107183.

15. Yu, S.-Z. (2016). Conventional HSMMs**To distinguish the conventional HSMMs from HMMs, we will call explicit duration HMM as "explicit duration HSMM," variable transition HMM as "variable transition HSMM," and residual time HMM as "residual time HSMM" in the rest of this book. Hidden Semi-Markov Models, 103-120. https://doi.org/10.1016/b978-0-12-8027677.00005-x.

16. Gao, J., Teng, D., \&Ertin, E. (2018). A Probabilistic Approach for Heart Rate Variability Analysis Using Explicit Duration Hidden Markov Models. 2018 IEEE Statistical Signal Processing Workshop (SSP). https://doi.org/10.1109/ssp.2018.8450781.

17. Dewar, M., Wiggins, C., \&amp; Wood, F. (2012). Inference in Hidden Markov Models with Explicit State Duration Distributions. IEEE Signal Processing Letters, 19(4), 235-238. https://doi.org/10.1109/1sp.2012.2184795. 